\title{
Cecum Schwannoma: Uncommon Colonic Mass and New Surgical Approach Using Single Site Laparoscopy
}

\author{
Virgilio George, Luis Velazco, Imtiaz A. Munshi, Bruce Robb, Bryan Holcomb, \\ Matthew Ziegler, Alyssa Fajardo \\ Department of Surgery, Indiana University School of Medicine, Indianapolis, USA \\ Email: vigeorge@iupui.edu
}

Received 17 January 2014; revised 16 February 2014; accepted 15 March 2014

Copyright (C) 2014 by authors and Scientific Research Publishing Inc.

This work is licensed under the Creative Commons Attribution International License (CC BY). http://creativecommons.org/licenses/by/4.0/

c) (i) Open Access

\begin{abstract}
Introduction: Schwannomas (SNs) are rare tumors derived from Schwann cells. They can occur almost anywhere, but the most common location is cranial nerve VIII where they present as acoustic neuromas. SNs rarely may be located in the GI tract. Case reports of stomach, small intestine, descending colon, and rectal SN exist. An extremely rare site is the ascending colon. Here, we present a case of a 45-year-old man with a diagnosed $S N$ of the ascending colon that was treated successfully via single site laparoscopic right hemicolectomy. Discussion: $S N s$ have been reported to have a wide range of clinical manifestations including intermittent gastrointestinal bleeding, intestinal obstruction, constipation, intussusception, and abdominal pain. The diagnosis is made by histopathological examination demonstrating KIT (CD117) and CD34 stained negative samples and $\mathrm{S}-\mathbf{1 0 0}$ protein is stained positive. Surgical resection is the mainstay of therapy.
\end{abstract}

\section{Keywords}

Schwannomas; Single Site Laparoscopic

\section{Introduction}

Schwannomas (SNs) are rare tumors derived from Schwann cells. The most common SN is an acoustic neuroma, located on cranial nerve VIII. Although historically the most common primary mesenchymal gastrointestinal (GI) tumors have been the gastrointestinal stromal tumors (GIST), SNs have occasionally been documented to grow from intestinal sources. In these cases, the stomach is the most common location [1]. SNs of the colon and rectum are rare, and only a small number of cases have been reported [1] [2]-[7]. The clinical presentation of these 
tumors varies with descriptions ranging from asymptomatic to abdominal pain and gastrointestinal hemorrhage [8]-[12]. The majority of these tumors behave as benign tumors, however the possibility of malignant degeneration has been reported and is associated with the size of the tumor. Surgical excision is the preferred method of treatment since the efficacy of both chemotherapy and radiotherapy remain uncertain [9].

Here, we present a case of a 45-year-old man with a diagnosed $S N$ of the ascending colon that was treated successfully by a single site laparoscopic right colectomy.

\section{Report of a Case}

The patient was a 45-year-old man, previously healthy, who developed bright red blood per rectum 2 weeks prior to presentation. He underwent colonoscopy and was found to have several small adenomatous polyps throughout the colon as well as a $20 \mathrm{~mm}$ sub epithelial mass on the ascending colon (Figure 1). Endoscopic ultrasound (EUS) was then obtained which reported one $20 \mathrm{~mm}$ nodule found in the mid ascending colon, which was felt to be compatible with extrinsic compression vs. GIST/or a tumor of mesenchymal origin. This area was not amenable to biopsy (Figure 2). A computed tomography (CT) scan of the abdomen and pelvis did not show metastatic disease or lymph node involvement. After the diagnostic work up, a colonoscopy for re-localization and tattooing was performed.

The patient then underwent laparoscopic, single site right hemicolectomy with ileo-colostomy. At the time of surgery, the specimen was opened in the operating room to confirm that the lesion was resected (Figure 3). His

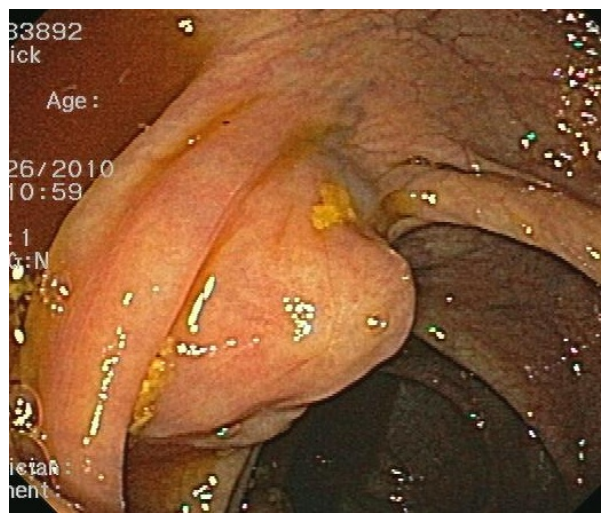

Figure 1. A $2 \mathrm{~cm}$ subepithelial mass was noted on colonoscopy at the level of the ascending colon.

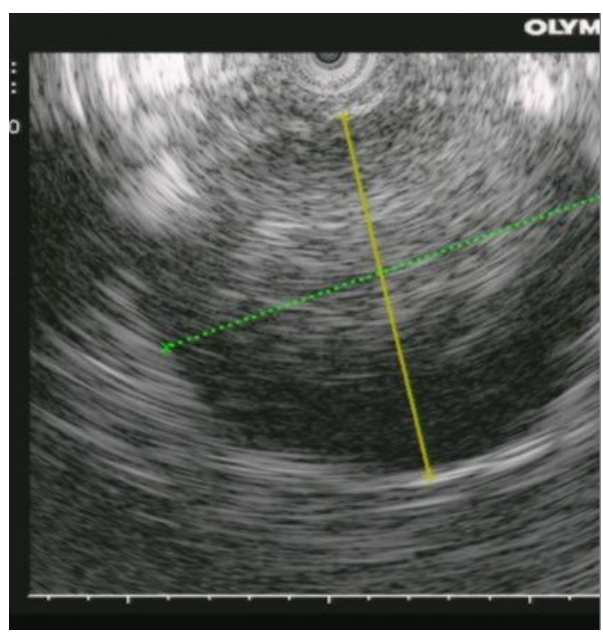

Figure 2. The $2 \mathrm{~cm}$ subepithelial mass seen on the endoscopic ultrasound. 


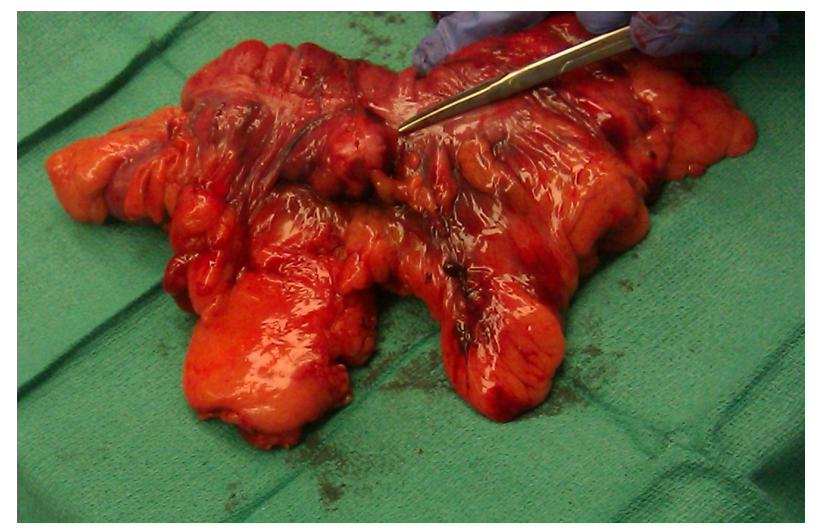

Figure 3. Right colon after laparoscopic resection showing the sub epithelia mass.

hospital course was uneventful and the patient was discharged on the third postoperative day.

The histopathological diagnosis of the specimen was consistent with cellular schwannoma, $2.8 \mathrm{~cm}$ in greatest dimension. The surgical margins were negative and there was no necrosis noted, and the mitotic count was less than 5/50 HPF. All the lymph nodes resected were negative for malignancy. The well-circumscribed lesion was contained within the submucosa and muscularis propia and was variably cellular. The nuclei were spindled and focal palisadings were identified. The periphery of the lesion had myxoid stroma. The overlying mucosa was intact and non-ulcerated. Immunohistochemical stains were performed and the lesion was positive for S100 (Figure 4) and negative for smooth muscle actin, CD117 (Figure 5), and CD34. B-catenin showed abundant background staining and is interpreted as negative.

\section{Discussion}

Tumors of mesenchymal origin of the GI tract can present in a wide variety of forms including as GISTs and SNs. Of these, GISTs are the most common and predominantly present in the stomach and small intestine [11]. $S N s$, on the other hand, have been rarely documented as primary gastrointestinal tumors. They are outnumbered by GIST by approximately 50-100:1 [1]. They may grossly and clinically resemble GISTs and most commonly occur in the stomach [11]. Only a handful of case reports describe the occurrence of SNs in the colon [2]-[11] [13]-[16]. Furthermore, we found only one case that was also managed by laparoscopic right colectomy reported in the United States [4]. Only a limited number of studies have described the histologic spectrum, clinico-pathologic profile, and differential diagnosis of this type of tumors [11] [17].

Miettinen et al. [11], in a large case study, found that this tumor does not have predilection for either sex and can present in a wide range of age (18 - 87) with a mean age of 65 years. Out of the 20 cases reported, seven were found in the cecum, six in the sigmoid and recto sigmoid colon, three in the transverse colon, two in the descending colon, one in the rectum, and none in the ascending colon. Prevot et al. [17], in a report of five cases, found the tumor was localized in the stomach in three cases, one in the esophagus, and only one was found in the ascending colon. SNs of the colon do not have any connection with Neurofibromatosis type 1 or type 2, as shown by several studies [11] [15] [17]. In our case, the patient was within the age range but the location was not characteristic.

SNs have been reported to have a wide range of clinical manifestations including intermittent rectal bleeding, intestinal obstruction, constipation, intussusception and chronic abdominal pain [8]-[12]. The diagnosis is considered to be definitive if the proliferation of spindle cell is histo-pathologically observed through HE staining and if both KIT (CD117) and CD34 are stained negative and S-100 protein is stained positive in immune histochemical staining [1] [17]. In our patient the CD117, CD34 negativity as well as S-100 positivity gave us the diagnosis.

Surgical resection is the mainstay of therapy. A tumor size of five $\mathrm{cm}$ is considered to be a criterion for surgery because recurrence and prognosis vary greatly if the tumor is larger. If a definitive preoperative diagnosis is possible, or if evidence of infiltration or metastasis is not observed in diagnostic imaging, the standard treatment is segmental colectomy, including laparoscopic surgery [18]-[20]. In our case, although the size of the tumor 


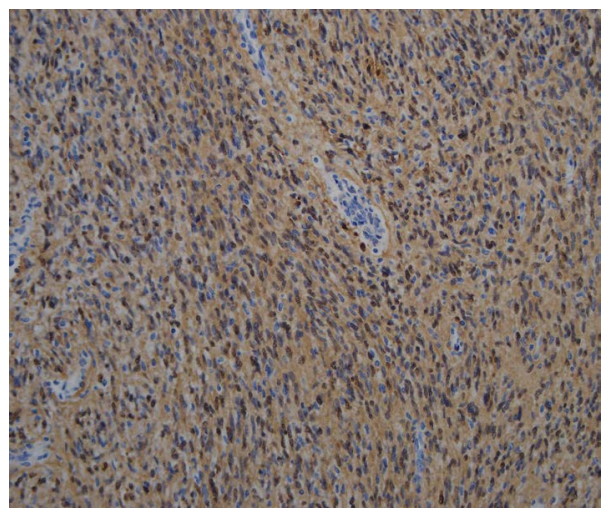

Figure 4. Immunohistochemical stain positive for protein S100.

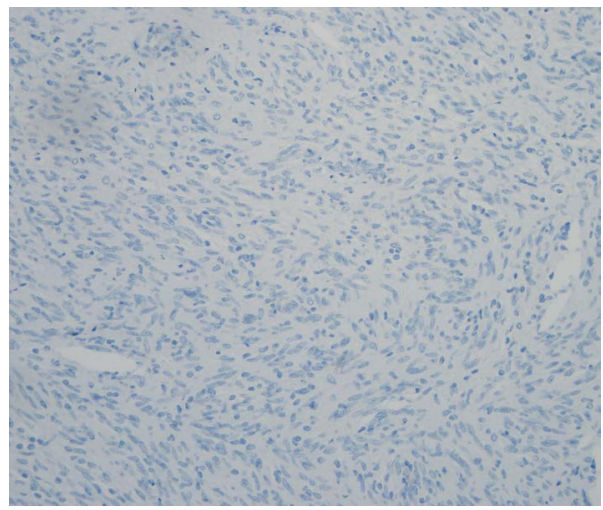

Figure 5. Immunohistochemical stain negative for CD117.

measured by colonoscopy and EUS was $<5 \mathrm{cms}$, surgery was performed for a definitive diagnosis. It should be noted that endoscopic approach has been used to resect this type of tumor in the past when the location is suitable [2] [3]. If the tumor is proved to be a schwannoma, this therapy is thought to be curative [9] [18].

\section{Conclusion}

We present the case of a 45-year-old man with a recent history of rectal bleeding who was found to have a mass in the ascending colon. He underwent surgical excision, and final pathology based on immune histochemical staining revealed a schwannoma. Ascending colon SNs are extremely rare, but should be included in the differential diagnoses of colonic masses. Surgical intervention is the treatment of choice for large lesions and if the lesion is not amenable to complete endoscopic resection.

\section{References}

[1] Markku, M. and Jerzy, L. (2001) Gastrointestinal Stromal Tumors, Definition, Clinical, Histological, Immunohistochemical, and Molecular Genetic Features and Differential Diagnosis. Virchows Archiv, 438, 1-12. http://dx.doi.org/10.1007/s004280000338

[2] Otake, Y., Abe, T., Otha, A., et al. (2001) A Case of Endoscopically Resected Schwannoma on the Sigmoid Colon. Progress in Digestive Endoscopy, 59, 118-119.

[3] Inagawa, S., Hori, M., Shimazaki, J., et al. (2001) Solitary Schwannoma of the Colon: Report of Two Cases. Surgery Today, 31, 833-838. http://dx.doi.org/10.1007/s005950170060

[4] Teitelbaum, E.N., Nguyes, S.Q., Zhu, H. and Salky, B.A. (2012) Laparoscopic Resection of a Scwannoma of the Ascending Colon. American Surgeon, 76, E97-E99.

[5] Ozawa, T., Watanabe, H., Okuyama, Y., Okumura, K., Tsuchiya, T., Tanji, N., Anzai, Y. and Unakami, M. (2001) A 
Case of Schwannoma of the Ascending Colon. Nihon Shokakibyo Gakkai Zasshi, 98, 167-173.

[6] Park, K.J., Kim, K.H., Roh, Y.H., Kim, S.H., Lee, J.H., Rha, S.H. and Choi, H.J. (2011) Isolated Primary Schwannoma Arising on the Colon: Report of Two Cases and Review of the Literature. Journal of the Korean Surgical Society, 80, 367-372. http://dx.doi.org/10.4174/jkss.2011.80.5.367

[7] Kim, H.J., Kim, C.H., Lim, S.W., et al. (2012) Schwannoma of the Ascending Colon Treated by Laparoscopic Right Hemicolectomy. World Journal of Surgical Oncology, 10, 81. http://dx.doi.org/10.1186/1477-7819-10-81

[8] Chris, B. and Nina, G. (2007) Schwannoma of the Colon Mimicking Carcinoma: A Case Report and Literature Review. International Journal of Colorectal Disease, 22, 1547-1548. http://dx.doi.org/10.1007/s00384-006-0264-9

[9] Constantine, F. and Ilias, K. (2005) Sigmoid Schwannoma: A Rare Case. World Journal of Gastroenterology, 11, 5079-5081.

[10] Melford, J. (1955) Neurilemmoma of the Sigmoid Colon, Associated with Diverticulitis as a Cause of Obstruction. American Journal of Surgery, 90, 139-142. http://dx.doi.org/10.1016/0002-9610(55)90672-3

[11] Markku, M. and Kris, S. (2001) Schwannomas in the Colon and Rectum. A Clinicopathologic and Immunohistochemical Study of 20 Cases. American Journal of Surgical Pathology, 25, 846-855. http://dx.doi.org/10.1097/00000478-200107000-00002

[12] Chihiro, T. and Hiroyuki, K. (2009) A Case of Bening Schwannoma of the Transverse Colon with Granulation Tissue. Case Reports in Gastroenterology, 3, 116-120. http://dx.doi.org/10.1159/000214837

[13] Adnan, M., Trikante, R. and Nejat, K. (2003) Schwannoma of the Iliocecal Valve: An Incidental Finding in a Patient with Rectal Carcinoma. American Journal of Gastroenterology, 98, S154.

[14] Mysorekar, V.V., Rao, S.G., et al. (2010) Schwannoma of the Ascending Colon. Indian Journal of Pathology and Microbiology, 53, 198-200.

[15] Daimaru, Y. and Kido, H. (1988) Benign Schwannoma of the Gastrointestinal Tract: A Clinicopathologic and Immunohistochemical Study. Human Pathology, 19, 257-264.

[16] Jacobson, B.C., Hirsch, M.S., Lee, J.H., et al. (2001) Multiple Asymptomatic Plexiform Schwannomas of the Sigmoid Colon. Gastrointestinal Endoscopy, 53, 801-804. http://dx.doi.org/10.1067/mge.2001.115317

[17] Prevot, S. and Bienvenu, L. (1999) Benign Schwannoma of the Digestive Tract: A Clinicopathologic and Immunohistochemical Study of 5 Cases, Including a Case of Esophageal Tumor. The American Journal of Surgical Pathology, 23, 431-436.

[18] Maciejewski, A., Lange, D. and Wloch, J. (2000) Case Report of Schwannoma of the Rectum Clinical and Pathological Contribution. Medical Science Monitor, 76, 779-782.

[19] Wilhem, D., Von Delius, S., Weber, L., Meining, A., Schenider, A., Friess, H., Schmid, R.M. and Frimberger, E. (2009) Combined Laparoscopic-Endoscopic Resections of Colorectal Polyps: 10-Year Experience and Follow Up. Surgical Endoscopy, 23, 688-693. http://dx.doi.org/10.1007/s00464-008-0282-5

[20] Pollock, J., Morgan, D., Denobile, J. and Williams, J. (2001) Adjuvant Radiotherapy for Gastrointestinal Stromal Tumor of the Rectum. Digestive Diseases and Sciences, 46, 268-272. http://dx.doi.org/10.1023/A:1005581000712 